\title{
Effect of pixel width, display width, and number of alternative signal locations on the detection of a simple vertical-line signal in visual noise
}

\author{
BERNARD MOULDEN and FRED KINGDOM \\ University of Reading, Reading, England
}

\begin{abstract}
Spatial resolution is one of the parameters that govern the visibility of digital displays. Three experiments are described, in which the effects of horizontal resolution and the interrelated parameters of pixel width and display width on the visibility of simple vertical-line signals in visual noise were investigated. A signal detection analysis of the results showed that detection efficiency increased substantially with pixel width, whereas an increase in display width and/or horizontal resolution had a very small, but significant, negative effect. The effect of pixel width is explained in terms of the contrast sensitivity function of the visual system. It is suggested that, in practical digital displays of the sort described, a system whereby selected portions of the input could be displayed using wider pixels would improve signal visibility.
\end{abstract}

There is a wide range of applications in which external events such as visual scenes, body scans, and underwater sounds are recorded digitally and then displayed as 2-D visual representations on a visual display unit (VDU). Often an operator is required to detect or recognize a target shape in the output display, and it is the responsibility of the engineer designing the system to maximize its visibility. The psychophysical investigation of digital displays can provide the engineer with useful information about various display parameters on operator performance, as well as provide theoretical information about the mechanics of visual processing. Both types of information emerge from the present investigation of the effect on signal detection performance of varying certain spatial parameters of a display consisting of a simple line signal in visual noise. This display constitutes a restricted but important class of digitized image; in a common instance, the horizontal axis of the display is used to represent frequency, the vertical axis to represent time, and the display intensity to represent amplitude, as in the familiar sound spectrograph.

Display "resolution" is generally defined as the number of pixels in the total width and height of the display; for example, a display with $512 \times 256$ resolution contains 512 columns and 256 rows of pixels. Resolution does not specify two other interrelated spatial parameters, pixel size and overall display size. One can expand these three parameters-resolution, pixel size, and display size-into

This research was funded by the Procurement Executive, Ministry of Defence. We would like to thank Richard Swensson of the Department of Radiology, Harvard Medical School, Boston, for his useful comments in the drafting of this manuscript. Requests for reprints should be sent to Bernard Moulden, Department of Psychology, Reading University, Whiteknights, Reading, Berks RG6 2AL, U.K. their six component parameters: in the "horizontal domain," (1) horizontal resolution (number of columns of pixels), (2) display width, and (3) pixel width; and in the "vertical domain," (4) vertical resolution (number of rows of pixels), (5) display height, and (6) pixel height. This paper investigates the three parameters in the horizontal domain, while holding constant those in the vertical domain. For example, if one holds pixel width constant, a change in horizontal resolution will necessarily result in a change in the width of the display. Accordingly, in each of three experiments, one horizontal display parameter was held constant while the remaining two were covaried. The effect on the visibility of a vertical straight-line signal was measured as a function of the varying parameters, and the results were analyzed to discover both the physical and perceptual effects of such parameters on signal detection.

In these experiments, $\boldsymbol{M}$-alternative forced-choice tasks were used to infer values of a detection index, $d^{\prime}$, for the signals employed. The size of $M$ ranged from 30 to 510 , a range substantially larger than is usually employed in signal detection experiments, where the range is invariably from 2 to 8 (Cohn \& Lasley, 1974; Swensson \& Judy, 1981; Swets \& Birdsall, 1978; though see Burgess \& Ghandeharian, 1984, for an exception to the rule, in which $M$ ranged from 2 to 1,800 ). High $M$ values offer the possibility of measuring performance using stronger signals than can be employed in the standard $M=1$ task. One issue of interest was whether the inferred values of $d^{\prime}$ would be reasonably consistent across the large range of $M$ employed, if all other factors were held constant.

The three experiments reported here are essentially variations upon a single theme, and will therefore be described together. 


\section{METHOD}

\section{Display Characteristics}

The stimuli were generated using a Supervisor 214 (Gresham Lion PPL) graphics/image system interfaced to a PDP-11/40 host computer and displayed on a Digivision TV monitor. The luminances of the $\mathbf{2 5 6}$ gray levels available in the system were measured using a United Detector Technology microphotometer, Model 81, focused onto the phosphor of the TV monitor when it displayed a large homogeneous patch of pixels.

The displays all contained 8 gray levels, or luminances, which were selected from the 256 available on the Supervisor 214 . The 8 luminances, which were set at equal logarithmic (ratio) intervals on the grounds that they would therefore be at roughly equal perceptual intervals, were $0.1,0.23,0.58,1.31,3.08,7.35,17.25$, and $40.58 \mathrm{~cd} / \mathrm{m}^{2}$.

Each pixel was randomly allocated one of the gray levels, $i$, with a probability, $P(i)$, determined by the binomial rule where

$$
P(i)=\frac{N !}{r !(N-r) !} p^{r}(1-p)^{N-r} .
$$

In this equation, $N=7$ and $r=i-1$. The underlying shape of the distribution of gray-level probabilities is determined by the parameter $p$ in the equation, where $p$ can range from 0 to 1 . When $p=0.5$, the distribution is symmetrical; as $p$ increases from 0.5 to 1 , the distribution becomes increasingly skewed to the left. The noise distribution was generated with $p_{\mathrm{n}}$ set to 0.5 . Figure la shows the resulting histogram of gray-level probabilities.

The mean luminance $\vec{L}_{\mathbf{n}}$ of the noise background was calculated numerically using the general formula:

$$
\bar{L}=\sum_{i} p_{i} L_{i}
$$

where $p_{i}$ and $L_{i}$ are, respectively, the probability of occurrence and luminance of the $i$ th gray level. It was found to be $3.78 \mathrm{~cd} / \mathrm{m}^{2}$.

The signals were generated with the $p$ value in the binomial distribution set to $p_{\mathrm{s}}>0.5$. In Experiment 1, for example, it was 0.56, and the resulting gray-level histogram is shown in Figure 1b. As can be seen from the figure, the proportion of pixels with higher luminances is greater in the signals than in the noise, which makes the signals perceptually distinguishable. For each of the three experiments, a slightly different signal strength was used. This was found to be necessary in order to ensure that subjects' detection rates fell roughly between $10 \%$ and $90 \%$ correct detections for all conditions. The $p_{s}$ values for the signals used in the three experiments, as well as their mean luminances, $\bar{L}_{\mathrm{s}}$, calculated as for the noise, are given in Table 1.

To make the displays as naturalistic as possible, the viewing distance was not tightly constrained. The nominal viewing distance was $120 \mathrm{~cm}$; in practice, subjects' head movements meant that the actual viewing distance varied between about 100 and $120 \mathrm{~cm}$. In view of this variation, we have given display dimensions in linear measure at the display surface, together with an indication of what these would mean in terms of visual angle at the nominal viewing distance of $120 \mathrm{~cm}$. Our tables and figures show visual angle, but it should be borne in mind that the values are approximations within the limits described above.

All displays were constructed from pixels that were approximately circular, with a diameter of $0.44 \mathrm{~mm}$ (equivalent to a visual angle of 1.27 ' of arc at $120 \mathrm{~cm}$ ). To construct "new" pixels of variable width, $w$, we simply allocated the same luminance to adjacent pixels to construct blocks of the appropriate size. The width of the basic display pixel was $0.44 \mathrm{~mm}$, and we constructed "new" pixels 1 , 2, 4, 8, and 16 times this width for use in Experiments 1 and 3.
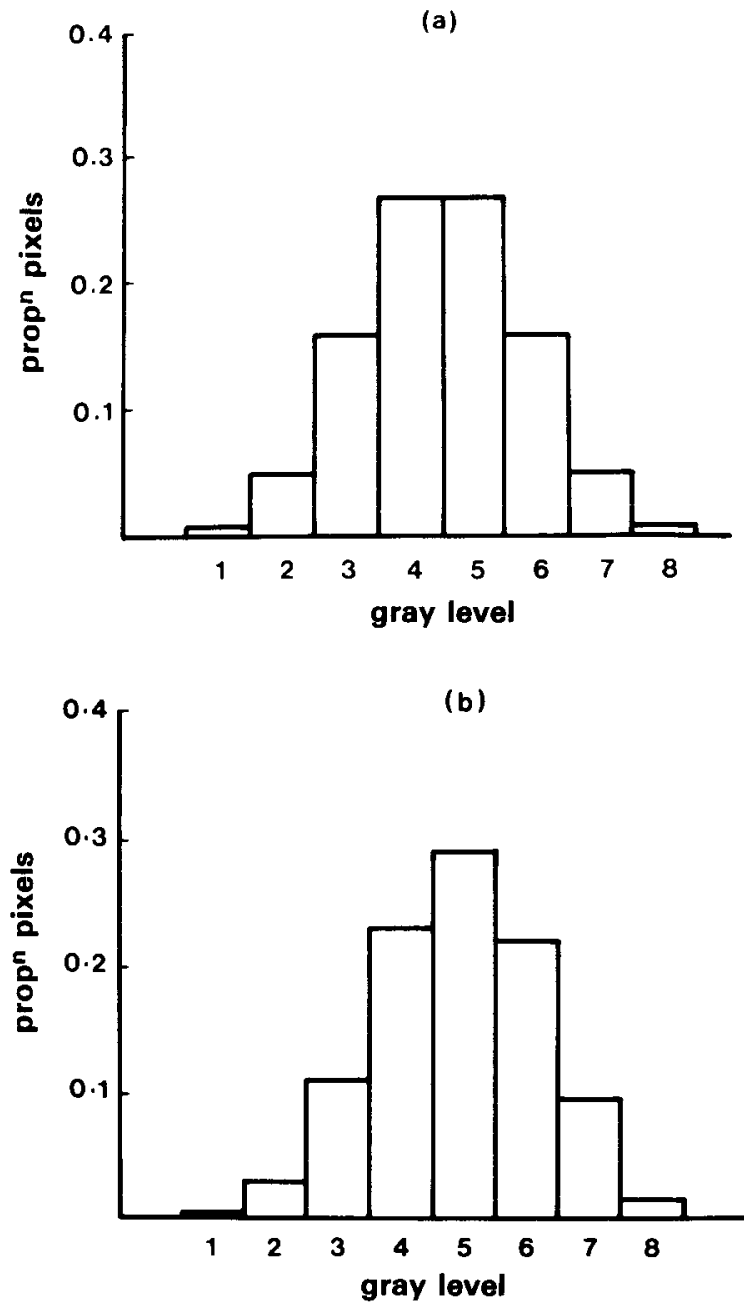

Figure 1. Gray-level histogram for (a) noise and (b) signals used in Experiment 1. The numbers along the abscissa are nominal and do not give the actual luminance values of the gray levels, which were $0.1,0.23,0.58,1.31,3.08,7.35,17.25$, and $40.58 \mathrm{~cd} / \mathrm{m}^{2}$. Because of the larger number of pixels with higher luminances, the signals were, on average, brighter than the noise.

The overall width of the display, $W$, varied with the experimental condition, as did also $M$, the number of alternative columns from which the signal had to be chosen. The values of $w, W$, and $M$ are all given, as appropriate, in the tables of results for each experiment.

For all conditions, the pixel height was always that of 1 (original) pixel, namely $0.44 \mathrm{~mm}$. The height of the noise background was 240 of these pixels, namely $10.56 \mathrm{~cm}$ (about $5.08^{\circ}$ of visual angle at $120 \mathrm{~cm}$ ). The display area above and below the noise background was filled to a total height of $22.53 \mathrm{~cm}$ with a homogeneous field whose luminance was that of the mean luminance of the noise.

The signals were always 1 "new" pixel wide. They extended the full height of the noise background and replaced one of the noise columns. Since the two columns of pixels on the extreme boundaries were not considered as candidates for signal locations, all the signals were surrounded by noise.

The noise backgrounds were produced in the following way. Using the appropriate computational values, an array of luminance values was freshly generated before each experimental session. This array 
Table 1

Measures of Signal Strength Used in the Three Experiments

\begin{tabular}{ccccccc}
\hline Experiment & $p_{s}$ & $\bar{L}_{s}$ & $\sigma_{\mathrm{s}}$ & $\sigma_{\mathrm{s}} / \sigma_{\mathrm{n}}$ & $S N R_{\mathrm{s}}$ & $S N R_{\mathbf{n}}$ \\
\hline 1 & 0.56 & 5.26 & 6.66 & 1.3 & 3.47 & 4.44 \\
2 & 0.57 & 5.55 & 6.97 & 1.4 & 3.42 & 5.31 \\
3 & 0.55 & 4.98 & 6.35 & 1.2 & 2.95 & 3.61 \\
\hline
\end{tabular}

Note-For the noise, $p_{\mathrm{n}}=0.5, \bar{L}_{\mathrm{n}}=3.78$, and $\sigma_{\mathrm{n}}=5.11$ for all experiments. $S N R=$ signal-to-noise ratio.

Table 2

Performance Measures as a Function of Pixel Width and the Number of Alternative Signal Locations for Experiment 1

\begin{tabular}{rrrrrrr}
\hline$w$ & $M$ & $P C$ & $d^{\prime}$ & $S E$ & $F \%$ & $S E$ \\
\hline 1.27 & 510 & 10.6 & 1.71 & 0.09 & 14.8 & 1.6 \\
2.54 & 254 & 27.8 & 2.19 & 0.06 & 24.4 & 1.4 \\
5.08 & 126 & 52.2 & 2.65 & 0.01 & 35.5 & 0.2 \\
10.16 & 62 & 75.5 & 3.12 & 0.2 & 49.7 & 6.5 \\
20.32 & 30 & 86.1 & 3.28 & 0.14 & 54.9 & 4.5 \\
\hline
\end{tabular}

Note $-w=$ pixel width in minutes of arc; $M=$ number of alternative signal locations; $P C=$ percentage of correct signal detections; $F=$ efficiency.

consisted of $3,072 \times 512$ pixels, each one representing the potential luminance value for a display pixel. These values were stored in the memory plans of the graphics generator. For each trial, a new $x \times 240$ block, where $x$ is the width of the display for that particular session, was randomly selected from this array to generate the noise background for that trial. A signal was then randomly selected from a set freshly generated before each sesion, and displayed with the noise as described above.

Plate 1 is a demonstration photograph of a display similar to those used in Experiment 1 (the strength of the signal shown here is of course much greater than that of the near-threshold signals actually used). The cursor positioned over the signal illustrates the method of detection.

\section{Subjects and Procedure}

Nine subjects were employed, 3 in each of the three experiments. All had normal vision and, with one exception, they were undergraduate students who had had no previous experience of experiments of this kind and were naive as to the purpose of the experiment. The single exception was F.K., one of the authors, who acted as a subject in Experiment 1.

The subject was first given a number of practice trials to ensure familiarity with the requirements of the experiment. During a single experimental session, the subject performed 60 trials, with all displays within a session having the same width characteristics. Each subject received three sessions for each of the experimental conditions.

During a trial, the subject was required to find the signal, defined as being the line that had, overall, the greatest intensity. He/she indicated the horizontal location of the signal by positioning a box cursor (see Plate 1) precisely over a noise or signal line by means of a tracker ball that he/she controlled. The size of the cursor was a function of the particular pixel-width condition. With the 1-pixelwide condition, the cursor was 3 pixels wide from edge to edge, the minimum available on the system. With the $w=x 2$-pixel-width condition, the cursor was 4 pixels wide. For the wider pixels, the cursor was the width of the "new" pixel. The subject pressed a button when satisfied with the choice and the response was recorded. For all conditions, if the subject erred by 1 (original) pixel in the positioning of the cursor over the signal, this was regarded as a location error and a "hit" was recorded. After the subject had made a response, the stimulus disappeared and, $4 \mathrm{sec}$ later, was replaced by another.

\section{Data Analysis}

Within-experiment. For each experimental condition and each subject, the percentage of correct signal detections $(P C)$ was calculated from the pooled results from the three sessions. These $P C$ data are given in Tables 2,3 , and 4 below.

The next stage of analysis was to convert the $P C$ measures into a $d^{\prime}$ measure, a signal detection theory (SDT) measure of sensitivity (Green \& Swets, 1966), for each subject and condition. We used an iterative search procedure to obtain $d^{\prime}$, using numerical evaluation of the following formula, which obtains proportion of correct signal detections $(\boldsymbol{P})$ from $d^{\prime}$,

$$
P=\int_{-\infty}^{+\infty} \Phi\left(x-d^{\prime}\right) \phi(x)^{M-1} d x .
$$

This estimation equation is the same as that used to derive the values of $d^{\prime}$ and $P C$ as a function of $M$ in the revised version of Elliot's (1964) tables produced by Hacker and Ratcliff (1979).

In the equation, $\phi$ and $\Phi$ represent the ordinate and area under the lower tail of the unit normal distribution and $M$ is the number of signal alternatives. The metric $d^{\prime}$ is therefore a measure of human sensitivity to a signal in noise in which the statistical effect of $M$ has been removed. That is not to imply that there is no perceptual effect of $M$ on performance in addition to the statistical effect. It may well be that as $M$ increases, there is a greater load imposed on the visual system in having to choose between so many alternatives, and one would expect $d^{\prime}$ to be a function of $M$ in this case. The results of any one experiment alone are not sufficient to measure the effect of $M$ on $d^{\prime}$ because of the confounding effect of $w$ (the pixel width). Taken together, however, the results of the three experiments make it possible to disambiguate the effect of $M$ from that of $w$. The metric $d^{\prime}$ is estimated on the assumption that the distribution of the perceptual variable that underlies the detection process is normally distributed, with an equal distribution for the signal and noise.

Between-experiment comparisons. To compare the results between experiments, the data needed to be normalized, since different signal strengths were used for each experiment (see Table 1). To normalize the data across experiments, we used a physical detection model that had been found in previous experiments (Kingdom \& Moulden, 1986; Moulden \& Kingdom, 1987) to give an

Table 3

Performance Measures as a Function of Display Width and Number of Alternative Signal Locations for Experiment 2

\begin{tabular}{rrrrlrl}
\hline \multicolumn{1}{c}{$W$} & $\boldsymbol{M}$ & $P C$ & $d^{\prime}$ & $S E$ & $F \%$ & $S E$ \\
\hline 0.68 & 30 & 64.3 & 2.47 & 0.09 & 22.0 & 3.5 \\
1.35 & 62 & 51.7 & 2.38 & 0.06 & 20.4 & 3.5 \\
2.71 & 126 & 37.7 & 2.24 & 0.01 & 18.0 & 3.1 \\
5.42 & 254 & 32.7 & 2.33 & 0.2 & 19.4 & 2.8 \\
10.83 & 510 & 20.0 & 2.12 & 0.14 & 16.2 & 2.9 \\
\hline
\end{tabular}

Note $-W=$ display width in degrees; $\boldsymbol{M}=$ number of alternative signal locations; $P C=$ percentage of correct signal detections; $F=$ efficiency.

Table 4

Performance Measures as a Function of Pixel Width and Display Width for Experiment 3

\begin{tabular}{ccccccc}
\hline$w$ & $W$ & $P C$ & $d^{\prime}$ & $S E$ & $F \%$ & $S E$ \\
\hline 1.27 & 0.68 & 31.2 & 1.50 & 0.2 & 16.8 & 2.3 \\
2.54 & 1.35 & 38.4 & 1.72 & 0.2 & 22.8 & 2.9 \\
5.08 & 2.71 & 51.1 & 2.08 & 0.19 & 33.8 & 5.8 \\
10.16 & 5.42 & 66.5 & 2.54 & 0.17 & 49.9 & 6.9 \\
20.32 & 10.83 & 69.9 & 2.64 & 0.19 & 53.7 & 5.7 \\
\hline
\end{tabular}

Note- $w=$ pixel width in minutes of arc; $W=$ display width in degrees; $P C=$ percentage of correct signal detections; $F=$ efficiency. 


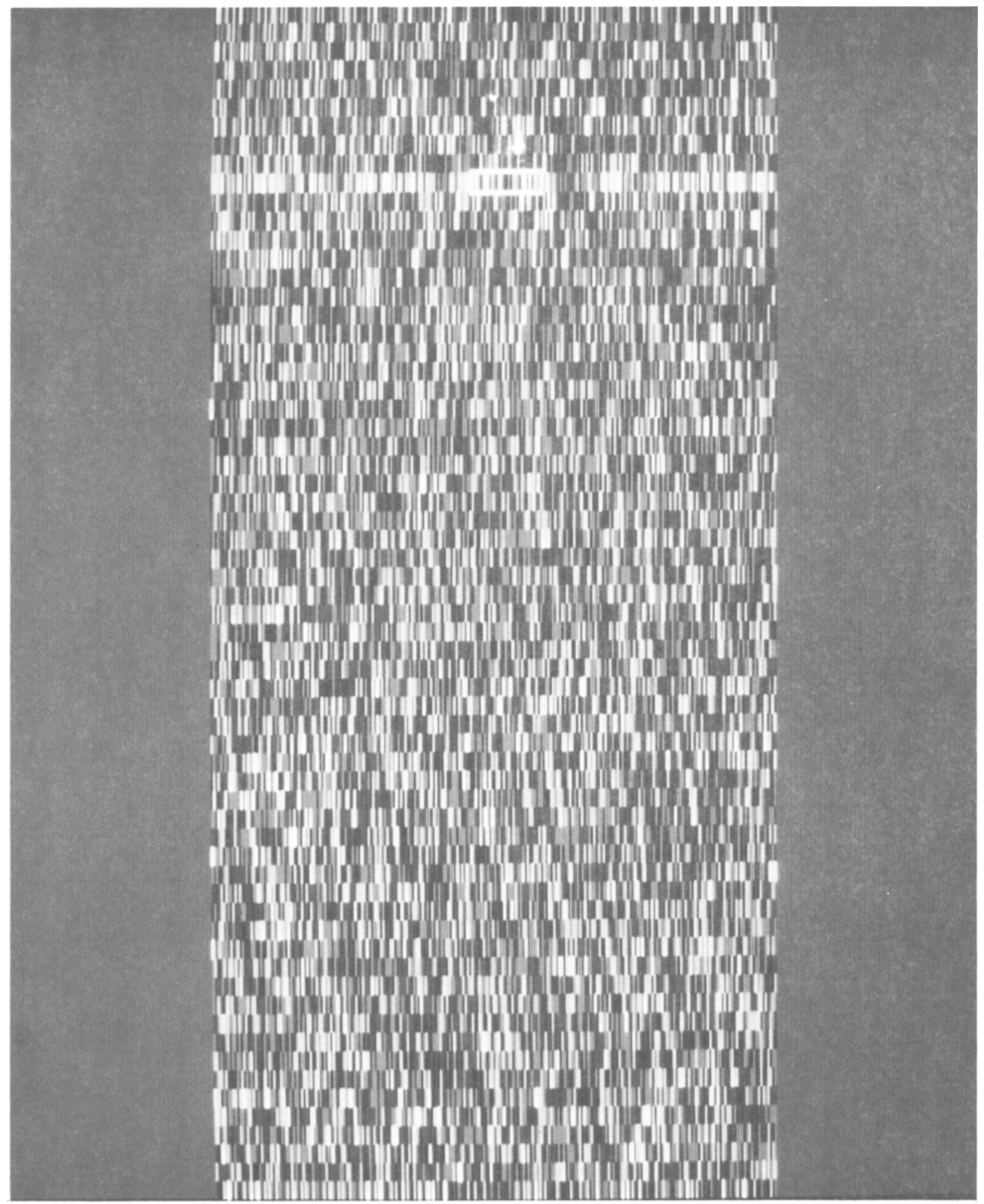

Plate 1. Demonstration photograph of the kind of display used in Experiment 1 . Pixel width, w, was 10.16' of arc. The strength of the signal shown here was much greater than that used in the actual experiment. The box cursor is located over the signal, indicating a correct detection. 
excellent description of detection performance with displays with widely different luminance characteristics (e.g., type of gray-level scale, number of gray levels, signal strength). The physical model may be described as an energy integrator model. It performs the detection task using a filter whose spatial dimensions match those of the signal. The output of the filter is proportional to the mean luminance of all the pixels that fall within it, and the assumption is that the filter is used as a traveling window to measure the average intensity of all the potential signal candidates in the display and to indicate as the signal the candidate with the highest value.

Two possible detection indices of the model detector are its signalto-noise ratio measured in units of either noise $\left(S N R_{n}\right)$ or signal $\left(S N R_{\mathbf{s}}\right)$ variance:

$$
\begin{aligned}
& S N R_{\mathrm{n}}=\frac{\left(\bar{L}_{\mathrm{s}}-\bar{L}_{\mathrm{n}}\right)}{\sigma_{\mathrm{n}} / \sqrt{N}} \\
& S N R_{\mathrm{s}}=\frac{\left(\bar{L}_{\mathrm{s}}-\bar{L}_{\mathrm{n}}\right)}{\sigma_{\mathrm{s}} / \sqrt{N}}
\end{aligned}
$$

$\bar{L}_{\mathrm{s}}$ and $\bar{L}_{\mathrm{n}}$ are the mean luminance of the signal and noise, respectively, $\sigma_{\mathrm{n}}$ and $\sigma_{\mathrm{s}}$ are the standard deviation of noise and signal pixel luminance, respectively, and $N$ is the number of pixels making up each potential signal location.

Table 1 gives the values of $S N R_{\mathrm{s}}$ and $S N R_{\mathrm{n}}$ computed for the signals used in the three experiments. The ratio of $S D$ s of the signals and noise $\sigma_{s} / \sigma_{n}$ are also presented, which gives the reciprocal of the slope of the $M=1$ ROC (receiver operating characteristic) curve for the model detector.

To normalize the data across experiments, we have computed the measure $F$, efficiency, using the index $S N R_{\mathrm{n}}$, where $F=\left(d^{\prime} / S N R_{\mathrm{n}}\right)^{2}$. Measures of efficiency are commonly used to compare human performance with that of an "ideal" detector, that is, one that is able to utilize all the statistical information in the display (Barlow, 1978; Barlow \& Reeves, 1979; Burgess \& Ghandeharian, 1984; Sullivan, 1983). Our model detector is not "ideal," since its performance is suboptimal, although its performance will correlate very highly with that of an ideal detector, as in fact it will with any simple physical model. In fact, the decision to use $S N R_{\mathrm{n}}$, as opposed to $S N R_{\mathrm{s}}$, to normalize the data in this way is an arbitrary one, and therefore it should be emphasized that no particular importance should be attributed to the absolute values of $F$.

\section{RESULTS}

\section{Experiment 1}

In this experiment, pixel width, $w$, and the number of alternative signal locations, $M$, were covaried, with $W$, the width of the display, being held constant at $22.5 \mathrm{~cm}$ (about $10.8^{\circ}$ ).

Inspection of Table 2 shows that detection rates improved rapidly at first with pixel $w$ (and/or decrease in $M$ ) and then leveled off. Figure 2 shows the normalized $F$ values for this experiment as the solid line. A two-way analysis of variance (ANOVA) with subjects and $w$ (or its covariate $M$ ) as factors shows a significant main effect of $w($ and/or $M)$ on $d^{\prime}[F(4,8)=29.6, p<.0001]$

\section{Experiment 2}

In this experiment, $M$, the number of alternative signal locations, and $W$, the overall width of the display, were covaried, while $w$, pixel width, was held constant at $1.27^{\prime}$ of arc.

Table 3 shows that $P C$ performance decreased as $W$ and $M$ increased, but that the $d^{\prime}$ values declined only slightly. A two-way ANOVA shows that the main effect of $W$ (and/or $M$ ) on $d^{\prime}$ is significant $[F(4,8)=4.9, p<.05]$. A straight line fitted to the data by a regression analysis, however, shows the decline in $d^{\prime}$ to be about $12 \%$.

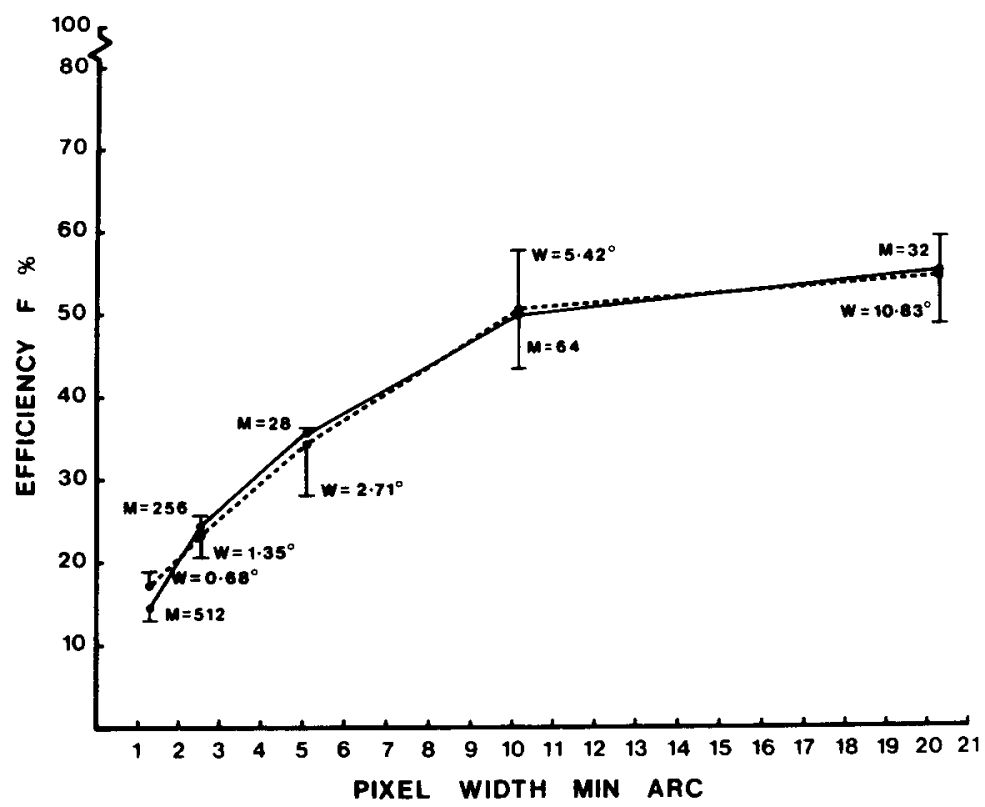

Figure 2. Mean efficiency, $F$, measures for Experiment 1 as a function of pixed width, $w$, and number of alternative signal locations, $M$ (continuous line), and for Experiment 3 as a function of pixel width, $w$, and display width, $W$ (dashed line). 
The reason for the slight decline in $d^{\prime}$ with $M$ and $W$ could be entirely attributed to the experimental method used. The subjects were scored for a correct detection if they positioned the cursor either directly on the line or on either of the two noise lines immediately adjacent to it. This may have resulted in a slight overestimation of the $P C$ results, since, on occasion, a subject may have been scored for a hit when in fact he/she had perceived the noise line adjacent to the signal as being the signal, instead of being scored for a "correct" hit made with a location miss. This slight overestimation will be greater for small $M$ than for large $M$, and would thus result in the slight decline of $d^{\prime}$ with $M$ and $W$, which was, indeed, found.

\section{Experiment 3}

In this final experiment, $W$, the width of the display, and $w$, pixel width, were covaried while $M$ was held constant at 32 .

Table 4 shows that both $P C$ and $d^{\prime}$ at first increased fairly rapidly as $w$ (and/or $W$ ) increased and then leveled off. The $F$ values for this experiment are shown as the dashed function in Figure 2, which also shows the results from Experiment 1. The two curves are plotted on the same graph to emphasize the near identity of the two sets of results. A two-way ANOVA with subjects and $w$ (or its covariate $W$ ) as factors shows a significant main effect of $w$ on $d^{\prime}$ (and/or $W$ ) $[F(4,8)=27.4, p<.0001]$.

\section{DISCUSSION}

The data from the first experiment show that detection performance increases with pixel width, $w$, and/or decreases with the number of alternative signal locations, $M$. If $M$ is having an effect on the $d^{\prime}$ measures, then the effect must be perceptual, since the statistical effect of $M$ on $d^{\prime}$ is taken into account in the latter's computation. It is most likely, however, that the change in performance across the conditions is principally due to the effect of $w$, since evidence from previous research suggests that the effect of $M$ on performance in visual SDT tasks can be accounted for entirely on statistical grounds (Burgess \& Ghandeharian, 1984; Swensson \& Judy, 1981). The results from the other two experiments support this hypothesis. The results of Experiment 2 imply that neither the number of alternative signal locations, $M$, nor the width of the display, $W$, have much effect on detection performance, once the statistical effect of $M$ has been removed. One would therefore expect that when $w$ is allowed to covary with $W$ (Experiment 3 ) as opposed to $M$ (Experiment 1), the results will be the same, and indeed they are, as the $F$ measures displayed in Figure 2 show. A simple correlation of the two sets of $F$ values gives $R=0.9$.

Why should detection rates improve with pixel width? The most likely explanation is that the visual system is less sensitive to the contrast of the vertical lines when the pixel widths are small than when they are large. It is well established that, with grating stimuli whose luminance is sinusoidally modulated, contrast sensitivity is a function of $S F$ (spatial frequency); sensitivity falls off at both high and low $S F$ s, with the peak of the $C S F$ (contrast sensitivity function) at around $2.0-5.0$ cycles per degree (cpd) (Campbell \& Robson, 1968). For square-wave data, there is found to be an even less rapid falloff at the low $S F$ end.

Each signal line of our displays could be considered as being one half-period of a grating whose spatial frequency is inversely proportional to the pixel width. The spatial frequencies would be about $1.5,3,6,12$, and $24 \mathrm{cpd}$ in order of increasing pixel width, and at first sight it might seem surprising that there is not a small decrease in detection efficiency for the widest-pixel condition $(w=$ $20.32^{\prime}$ of arc, equivalent to a half-period of a 1.5 -cpd grating). However, this is not so surprising when one considers the power spectrum of our stimuli in more detail. Unlike an infinitely extended grating, a single half-period has a continuous spectrum, and although the spectra of lines of varying width differ in terms of their energy at higher spatial frequencies, they are very similar in terms of their power at low spatial frequencies. If it is this lowfrequency energy that principally determines detection, this would explain the absence of a decline in detection efficiency for the largest pixel widths. It should also be pointed out that the "new" pixels of various widths used here may have slightly sharper edges than the smallestwidth pixels from which they are constructed. If so, the wider pixel displays will have greater power in the high frequencies, and this may be partly responsible for their greater signal visibility. A related point is that if the edges of the thinnest pixels are indeed more diffuse, it may be more difficult for the subject to construct an internal vertical "ruler" to allow for accurate vertical integration of luminance over a whole line. These possible explanations of the results need to be reexamined when information about the spectral composition of the various pixel-width displays has been obtained. We hope to carry out the necessary investigations in the near future.

\section{CONCLUSIONS}

The results of this investigation have shown that, with displays such as the ones used here, the detectability of vertical-line signals is affected by certain spatial dimensions of the display, in this case pixel width, but not by others, such as display width and horizontal resolution. On the other hand, we have shown in a number of studies (Kingdom \& Moulden, 1986; Moulden \& Kingdom, 1987) that changes in the luminance characteristics of the display (e.g., number of gray levels, type of gray scale, mean luminance, range and contrast) have no significant effect on such detection once the data have been normalized with respect to a simple physical model of detection.

What practical value do the results of this investigation have for display technology? For a display in which vertical lines are required to be detected in visual noise, such as the time versus frequency displays used to display underwater sounds, it might well be advantageous to have 
a "window" that can redisplay sections of the input using wider pixels for closer inspection.

The next main stage in our research is to investigate the effect on detection rates of the vertical domain parameters described in the introduction."

\section{REFERENCES}

BARLOW, H. B. (1978). The efficiency of detecting changes of density in random dot patterns. Vision Research, 18, 637-650.

BARLOW, H. B., REEVES, B. C. (1979). The versatility and absolute efficiency of detecting mirror symmetry in random dot displays. $\mathrm{Vi}$ sion Research, 19, 783-793.

Burgess, A. E., Ghandeharian, H. (1984). Visual signal detection: II. Signal-location identification. Journal of the Optical Society of America, 1, 906-910.

Campbell, B. F., Robson, J. G. (1968). Application of Fourier analysis to the visibility of gratings. Joumal of Physiology, 197, 551-563.

CoHN, T. E., \& LASLEY, D. J. (1974). Detectability of a luminance increment: Effect of spatial uncertainty. Journal of the Optical Society of America, 64, 1715-1719.

Elliot, P. B. (1964). Tables of $d^{\prime}$. In J. A. Swets (Ed.), Signal detection and recognition by human observers. New York: Wiley.

Green, D. M., \& Swets, J. A. (1966). Signal detection and psychophysics. New York: Wiley.
HaCker, M. J., \& RatclifF, R. A. (1979). A revised table of $d^{\prime}$ for M-alternative forced choice. Perception \& Psychophysics, 26, 168-170.

Kingdom, F., \& Moulden, B. (1986). Digitized images: What type of grey scale should one use? Perception, 15, 17-25.

MouldEN, B., \& KingDOM, F. (1987). Effect of the number of grey levels on the detectability of a simple line signal in visual noise. Spatial Vision, 2, 61-77.

Sullivan, G. D. (1983). Perceptual filters. In O. J. Braddick \& A. C. Sleigh (Eds.), Physical and biological processing of images. Berlin: Springer-Verlag.

SWENSSON, R. G., Judy, P. F. (1981). Detection of noisy visual targets: Models for the effects of spatial uncertainty and signal-to-noise ratio. Perception \& Psychophysics, 29, 521-534.

SweTs, J. A., \& Birdsall, T. G. (1978). Repeated observation of an uncertain signal. Perception \& Psychophysics, 23, 269-274.

\section{NOTE}

1. Note addeded in proof: Now published as Moulden, B., \& KINGDOM, F. (1987). Effect of pixel height, display height, and vertical resolution on the detection of a simple vertical line signal in visual noise. Human Factors, 29, 433-445.

(Manuscript received August 4, 1986; revision accepted for publication December 3, 1987.) 